\title{
Effects of a localized beam on the dynamics of excitable cavity solitons
}

\author{
Adrian Jacobo, ${ }^{*}$ Damià Gomila, ${ }^{\dagger}$ Manuel A. Matías, ${ }^{\ddagger}$ and Pere Colet ${ }^{\S}$ \\ IFISC, Instituto de Física Interdisciplinar y Sistemas Complejos (CSIC-UIB), E-07122 Palma de Mallorca, Spain
}

(Received 15 August 2008; published 19 November 2008)

\begin{abstract}
We study the dynamical behavior of dissipative solitons in an optical cavity filled with a Kerr medium when a localized beam is applied on top of the homogeneous pump. In particular, we report on the excitability regime that cavity solitons exhibit which is an emergent property since the system is not locally excitable. The resulting scenario differs in an important way from the case of a purely homogeneous pump and now two different excitable regimes, both class I, are shown. The whole scenario is presented and discussed, showing that it is organized by three codimension- 2 points. Moreover, the localized beam can be used to control important features, such as the excitable threshold, improving the possibilities for the experimental observation of this phenomenon.
\end{abstract}

DOI: 10.1103/PhysRevA.78.053821

PACS number(s): 42.65.Tg, 42.65.Sf, 05.45.-a, 89.75.Fb

\section{INTRODUCTION}

Dissipative solitons (also known as localized structures) are states in extended media that consist of one (or more) region(s) in one state surrounded by a region in a qualitatively different state (in the following this surrounding state is an area in a stable stationary state). These structures were first suggested in Refs. [1,2] and then described in a variety of systems, such as chemical reactions [3], semiconductors [4], granular media [5], binary-fluid convection [6,7], vegetation patterns [8], and also in nonlinear optical cavities where they are usually referred to as cavity solitons (CS) [9-13] (see Ref. [14-16] for recent surveys). Their potential in optical storage and processing of information has been stressed [17]. In this work we shall consider solitons that appear in a subcritical pattern forming bifurcation [11,18,19].

In general, dissipative solitons may develop a number of instabilities like start moving, breathing, or oscillating. In the latter case, they would oscillate in time while remaining stationary in space, like the oscillons (oscillating localized structures) found in a vibrated layer of sand [5]. The occurrence of these oscillons in autonomous systems has been reported both in optical [20,21] and chemical systems [22]. It has been shown that they can become unstable leading to excitable solitons in systems for which the local dynamics is not excitable $[23,24]$. In this case excitability appears as an emergent property arising from the spatial dependence, which allows for the formation of these structures. In particular, for solitons arising in uniformly pumped Kerr cavities, excitability is mediated by a saddle-loop (homoclinic) (SL) bifurcation, and it is characterized by a large excitability threshold and by occurring at any point of space that is properly excited $[23,24]$.

Since CS excitability emerges from the spatial dependence it is interesting to study the effect of breaking the

\footnotetext{
*jacobo@ifisc.uib-csic.es

†damia@ifisc.uib-csic.es

†manuel.matias@ifisc.uib-csic.es

$\S$ pere@ifisc.uib-csic.es

"http://ifisc.uib-csic.es
}

translational symmetry on the excitable dynamics. In optical systems this can be easily done by applying a (small amplitude) localized beam on top of the homogeneous pump. Addressing beams are typically used already to create CS by applying a transient perturbation. Here we analyze the dynamics of CS in a Kerr cavity where we apply a permanent addressing beam. On the one hand, this pump allows us to control the place where a CS appears. On the other hand, the system remains excitable and a new route, mediated by a saddle-node on an invariant circle (SNIC) bifurcation, appears. It is characterized by the fact that the excitable threshold is fully tunable, as it scales with the proximity to the bifurcation.

This paper is organized as follows. The model and overall dynamical behavior exhibited by the system in parameter space are described in Secs. II and III. Section IV addresses the instability exhibited by CS through a SNIC bifurcation. Section V discusses the excitable routes found in this system. Sections VI and VII discuss the codimension-2 points that organize the overall scenario. Finally, concluding remarks are given in Sec. VIII.

\section{MODEL}

A prototype model describing an optical cavity filled with a nonlinear Kerr medium is the one introduced by Lugiato and Lefever [25] with the goal of studying pattern formation in this optical system. Later studies showed that this equation also exhibits CS in some parameter regimes [20,26]. The model describes the dynamics of the slowly varying amplitude of the electromagnetic field $E(\vec{x}, t)$ in the paraxial and mean-field approximations $[\vec{x}=(x, y)$ is the plane transverse to the propagation direction $z$ on which the slow dynamics takes place]. The dynamics of the field is given by

$$
\frac{\partial E}{\partial t}=-(1+i \theta) E+i \nabla^{2} E+E_{I}(\vec{x})+i\left|E^{2}\right| E,
$$

where $\nabla^{2}=\partial^{2} / \partial x^{2}+\partial^{2} / \partial y^{2}$.

The first term on the right-hand side describes cavity losses, $E_{I}(\vec{x})$ is the input field (pump), $\theta$ is the cavity detuning with respect to $E_{I}$, and the sign of the cubic term repre- 
sents the self-focusing case. Notice that in the absence of losses and of an input field, the field can be rescaled to $E$ $\rightarrow E e^{i \theta t}$ to remove the detuning term and Eq. (1) becomes the nonlinear Schrödinger equation (NLSE). It is well documented that in this case, and in two spatial dimensions, the NLSE exhibits the so-called collapse regime [27], in which energy accumulates at a point of space. Collapse is prevented in Eq. (1) by the cavity losses leading to stable CS.

For spatially homogeneous pump $E_{I}(\vec{x})=E_{0}$, Eq. (1) has a homogeneous steady state solution given implicitly by $E_{0}$ $=E_{s}\left[1+i\left(\theta-I_{s}\right)\right]$, where $I_{s}=\left|E_{s}\right|^{2}[25]$. This solution is stable for low pump strength, that is for $I_{s}<1$. At $I_{s}=1$, the socalled modulation instability (MI) point, the homogeneous solution becomes unstable and extended patterns appear subcritically. The patterns arising at MI are typically oscillatory and increasing the pump they undergo further instabilities which eventually lead to optical turbulence [28,29]. Static hexagonal patterns can be found subcritically, that is, decreasing the pump value below the MI point. CS appear in the region of bistability between the homogeneous solution and the pattern. In fact there are two CS that appear through a saddle-node (fold) bifurcation, the one with larger amplitude (upper-branch CS) is stable at least for some parameter range, while the one with smaller amplitude (middle-branch $\mathrm{CS}$ ) is always unstable. Early studies already identified that the upper branch CS may undergo a Hopf bifurcation leading to an oscillatory behavior [20]. The oscillatory instabilities, as well as azimuthal instabilities, were fully characterized later [21]. As one moves in parameter space away from the Hopf bifurcation, the CS oscillation amplitude grows, and finally the limit cycle touches the middle-branch CS in a saddle-loop bifurcation which leads to a regime of excitable dissipative structures [23,24].

Here, we consider a pump beam of the form

$$
E_{I}(r)=E_{0}+H \exp \left(-r^{2} / r_{0}^{2}\right),
$$

where $E_{0}$ is a homogeneous field, assumed real, $H$ is the height of the localized Gaussian perturbation, $r^{2}=x^{2}+y^{2}$ and $r_{0}$ is the width of the Gaussian. For convenience, we write the height of the Gaussian beam as

$$
H=\sqrt{\left(I_{s}+I_{s h}\right)\left[1+\left(\theta-I_{s}-I_{s h}\right)^{2}\right]}-E_{0},
$$

where $I_{s}$ is the background intracavity intensity (due to $E_{0}$ ) and $I_{s}+I_{s h}$ corresponds to the intracavity field intensity of a cavity driven by an homogeneous field with an amplitude equal to one at the top of the Gaussian beam, $E_{I}=E_{0}+H$. This directly relates the height of the Gaussian beam $H$ with the equivalent intracavity intensity for a homogeneous pump. Notice that for $I_{s h}=0$ the pump beam becomes homogeneous, $H\left(I_{s h}=0\right)=0$. With the inclusion of the localized pump beam the system has now three independent control parameters which for convenience take as the background intensity, $I_{s}$, the detuning $\theta$, and $I_{s h}$.

Throughout this paper we will consider $r_{0}=1$. This corresponds to a Gaussian perturbation with a size smaller than the typical soliton size. Localized pumps of width larger than that of a soliton may lead to the presence of multiple structures or azimuth instabilities which will eventually evolve to an extended pattern. For localized pumps of width smaller

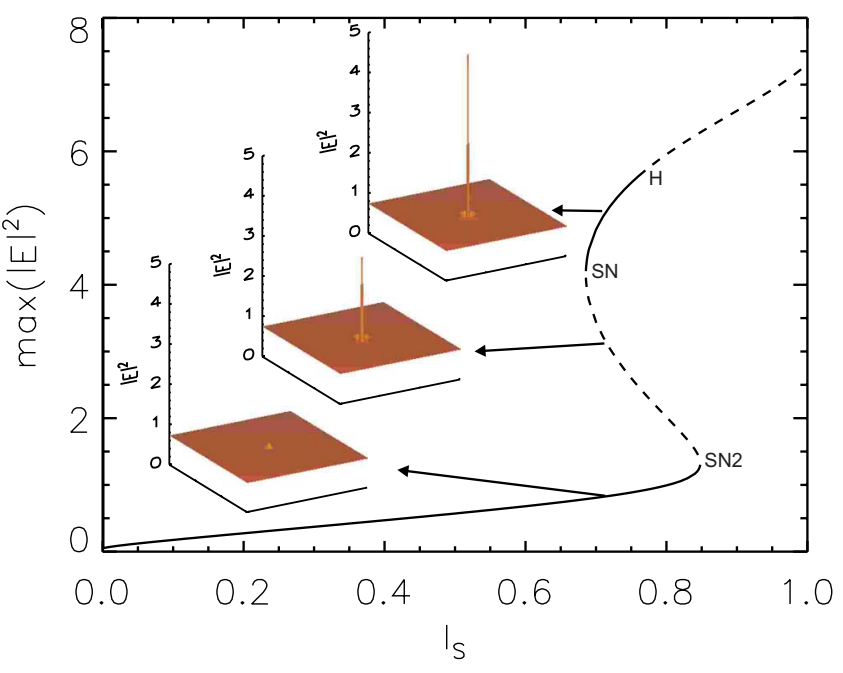

FIG. 1. (Color online) Bifurcation diagram, $\max (I)$ vs $I_{s}$, for a CS for $I_{s h}=0.3, \theta=1.25$. Solid lines represent stable solutions and dashed lines unstable ones. The insets show the transverse profile of the solutions. All the quantities plotted in this figure as well as in the other figures of this paper are dimensionless.

than the soliton, we have observed that a small change in the beam width leads to similar results as the ones shown here for $r_{0}=1$; in fact changing $r_{0}$ is similar to changing $I_{s h}$. The most relevant parameter is not the width or the height but its total energy. Thus, for a fixed $r_{0}, I_{s h}$ measures the energy of the Gaussian perturbation.

The numerical methods used to study this system are detailed in the Appendix of Ref. [24]. Equation (1), with the applied pump (2), has been solved numerically using a pseudospectral method, where the linear terms are integrated exactly in Fourier space, while the nonlinear ones are integrated using a second order in time approximation. Periodic boundary conditions in a square lattice of size $512 \times 512$ points were used. The stability of steady CS has been studied using the semianalytical method discussed in the abovementioned Appendix [24], using the radial version of Eq. (1), that simplifies the study taking into account the axisymmetric nature of the solutions.

\section{OVERVIEW OF THE BEHAVIOR OF THE SYSTEM}

One of the main consequences of the application of a localized pump is the breaking of the translational symmetry of Eq. (1). Solutions are now pinned in the region in which the Gaussian pump is applied. This also affects the transverse profile of the solutions, in particular the fundamental solution is no longer spatially homogeneous but it exhibits a bump as illustrated by the lower inset in Fig. 1.

To better understand the effects of the application of a localized pump, a diagram such as the one shown in Fig. 2 of Ref. [24], that represents the maximum intensity of the transverse field as a function of $I_{s}$ is shown in Fig. 1, namely for $I_{s h}=0.3$ and $\theta=1.25$. The diagram, with three branches, looks qualitatively equivalent to the case of a homogeneous pump and operations such as switching on and off the CS can be performed in a similar way. For example, with the system at 


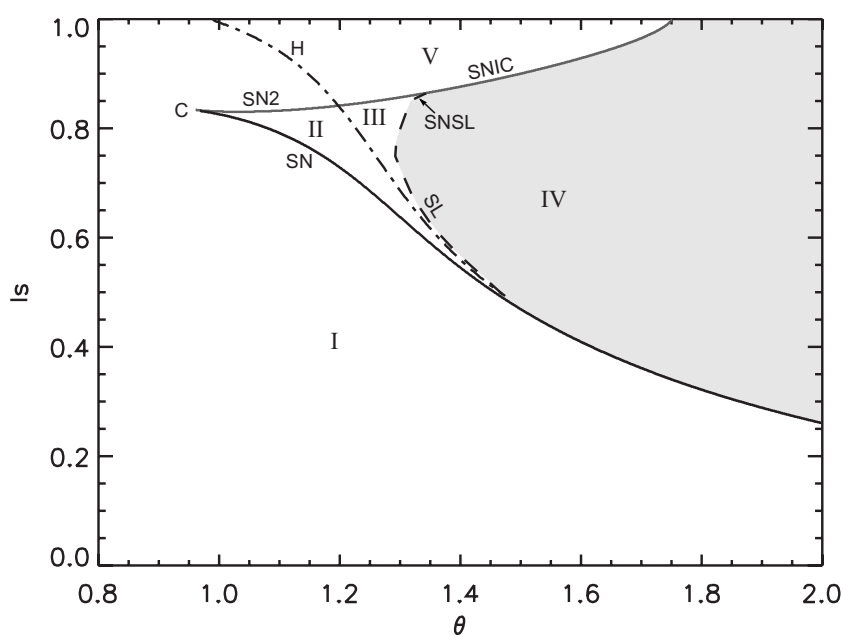

FIG. 2. Two-parameter $I_{s}$ vs $\theta$ phase diagram for $I_{s h}=0.3$. Bifurcation lines are SN (saddle-node); H (Hopf); SL (saddle-loop); SNIC (saddle-node on the invariant circle); SN2 (saddle-node off invariant cycle). Regions delimited by bifurcation lines are as follows. I, only the fundamental solution is stable; II, stationary stable CS coexisting with the fundamental one; III, oscillating CS (or oscillons), coexisting with the fundamental solution; IV, excitable region; V, oscillating CS (with no other coexisting solution).

the fundamental solution, the upper branch CS can be switched on by applying an additional transient localized beam or equivalently by temporarily increasing $I_{s h}$.

A relevant difference is that for a homogeneous pump the lowest branch (homogeneous solution) extends until the MI at $I_{s}=1$, while in the case considered here the fundamental solution merges with the middle branch CS before the MI, in a saddle-node bifurcation (that happens at $I_{s}=0.8479$, SN2 point in Fig. 1). To understand qualitatively this phenomenon one must take into account that the homogeneous pump case has many symmetries, some of which are broken when a localized pump is applied. In technical parlance one says that the bifurcation has become imperfect (see, e.g., [30]), with the consequence that a gap in $I_{s}$ appears making the lower branch disconnected in two branches (the right-hand part of the branch is not plotted in Fig. 1 and correspond to solutions unstable to extended patterns).

Exploring now the upper branches, in Fig. 1 past the saddle-node bifurcation at $I_{s}=0.6857$ ( $\mathrm{SN}$ point), a pair of stationary (stable, upper branch, and unstable, middle branch) localized solutions in the form of CS are found. In this parameter region, these structures are not essentially different to the solutions found in the homogeneous case [24]. Increasing $I_{s}$ the stable high-amplitude CS undergoes a Hopf bifurcation.

Overall the scenario found for a localized pump displays a richer variety of dynamical behaviors than in the case of homogeneous pump. Figure 2 shows a phase diagram for a fixed value of the localized pump $I_{s h}=0.3$. One can compare this figure with Fig. 1 in Ref. [24], corresponding to $I_{s h}=0$. The effect of breaking the translational symmetry would be to unfold some of the lines at $I_{s}=1$ (not visible in Fig. 1 of Ref. [24]), that are degenerate with the MI line, and also make the SN line end at $I_{s}<1$ (point $C$ in Fig. 2). Thus, the

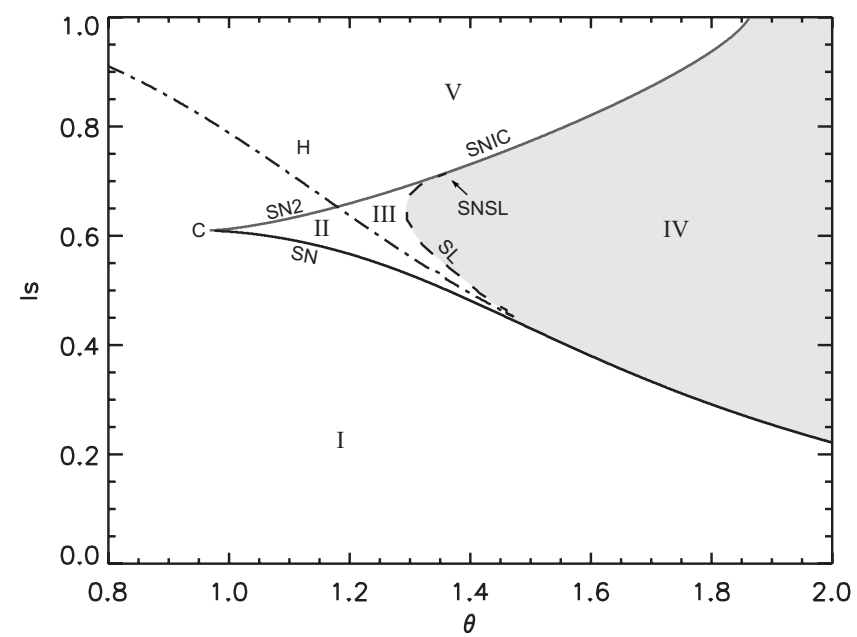

FIG. 3. Two-parameter $I_{s}$ vs $\theta$ phase diagram for $I_{s h}=0.7$. Line and region labeling as in Fig. 2.

effect of a localized pump is to push down the SN2-SNIC line (to be explained later), as is clear from Fig. 3, that provides a similar plot for $I_{s h}=0.7$.

Some of the most prominent features of these figures, in comparison with the homogeneous pump case, associated to the appearance of the SNIC line (to be discussed in more detail in Sec. IV) are that the excitable region, IV in Figs. 2 and 3 can have two types of excitable behavior (see Sec. V), both of class I, as two different transitions to oscillatory behavior are possible, saddle loop (SL) and SNIC. In addition, one has a new region, $\mathrm{V}$, in which one has a single attractor in the system, that is oscillatory, to be distinguished from region III, in which the system exhibits bistability [31], between the (stationary) fundamental solution and an oscillatory upper branch CS. These behaviors, and how they are organized by three codimension-2 points will be the subject of Secs. VI and VII.

\section{SADDLE-NODE ON THE INVARIANT CIRCLE BIFURCATION}

A saddle-node on the invariant circle bifurcation (SNIC), also known as saddle-node infinite-period (SNIPER) and as saddle-node central homoclinic bifurcation, is a special case of a saddle-node bifurcation that occurs inside a limit cycle. Although this bifurcation is local in (one-dimensional) flows on the circle, it has global features in higher-dimensional dynamical systems [30], so it is also termed local global or semilocal. In particular, the (stable) manifolds of the saddle and node fixed points transverse to the center manifold are organized by an unstable focus inside the limit cycle. At one side of the bifurcation the system exhibits oscillatory behavior, while at the other side the dynamics of the system is excitable. This mechanism leading to excitability has been found in several (zero dimensional) optical systems [32-34].

When approaching the bifurcation from the oscillatory side the period lengthens and becomes infinite. Quantitatively the period as a function of a parameter exhibits a inverse square root singular law [30], 


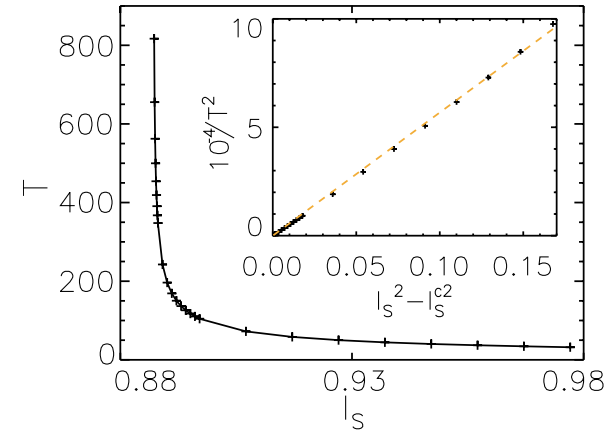

FIG. 4. (Color online) Period of the limit cycle $T$ as a function of $I_{s}$ for $I_{s h}=0.3$ and $\theta=1.45$. Inset: $1 / T^{2}$ vs $I_{s}$ close to the bifurcation point.

$$
T \propto\left[I_{s}^{2}-\left(I_{s}^{c}\right)^{2}\right]^{-1 / 2} .
$$

This can be used to distinguish the SNIC from other bifurcations leading to oscillatory behavior (e.g., from the saddleloop bifurcation with a logarithmic singular law $[23,24,30])$. Figure 4 shows the period of the oscillations as a function of $I_{s}$ obtained by numerical simulations of Eq. (1). In the inset of Fig. 4 we plot $1 / T^{2}$ versus $I_{s}^{2}$ close to the bifurcation point. The linear dependence obtained corroborates the scaling law and that the transition takes place through a SNIC bifurcation.

The overall route exhibited by the system along a vertical cut in Fig. 2 at $\theta=1.45$ is illustrated in Fig. 5, as the parameter $I_{s}$ is decreased from the top to the bottom of the figure. Thus, the figure shows the transition from oscillatory behavior (top panel) to stationary (fourth panel) through the occur-

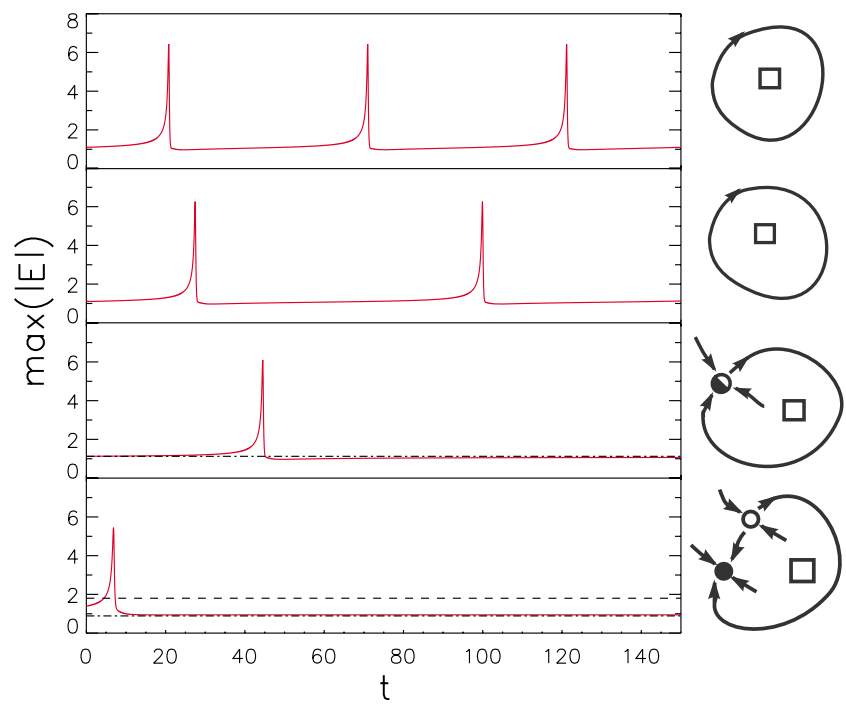

FIG. 5. (Color online) Time evolution of the CS amplitude for $I_{s h}=0.3, \theta=1.45$ and decreasing values of $I_{s}$. From top to bottom, $I_{s}=0.927,0.907,0.8871,0.8$. In the bottom panel the dashed line corresponds to the amplitude of the unstable CS (saddle) while the dotted-dashed line corresponds to the stable fundamental solution. These two solutions coincide when the SNIC bifurcation takes place (third panel). The sketches on the right-hand side illustrate the phase space dynamics.

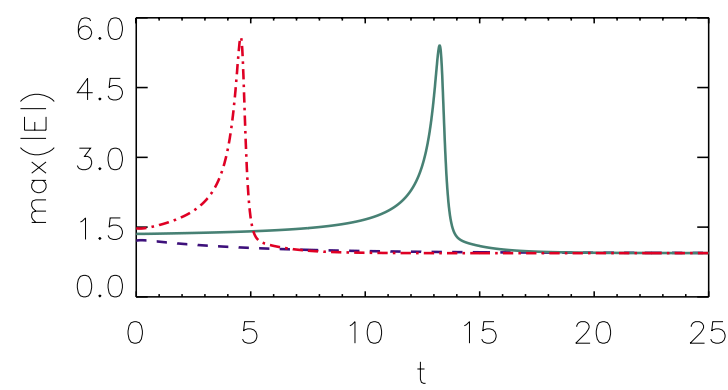

FIG. 6. (Color online) Evolution of the maximum of the field amplitude after applying a localized perturbation to the fundamental solution. The perturbation has the shape of the unstable stationary CS (saddle), that is scaled by 0.95 (blue dashed line), 1.01 (green solid line), and 1.1 (red dotted line). Here $I_{s h}=0.3, I_{s}=0.8$, and $\theta$ $=1.45$.

rence of a SNIC bifurcation (third panel). Lengthening of the period can be seen in the second panel. The sketches shown on the right-hand column of Fig. 5 illustrate the structure of the phase space. The validity of this scenario is further reinforced with the quantitative analysis presented in Sec. VII.

\section{EXCITABILITY}

An interesting aspect of this scenario is that in region IV one can have excitable behavior through two different mechanisms. On the one hand, and similarly to the behavior analyzed in Refs. [23,24], close enough to the SL line one has excitability if the fundamental solution is appropriately excited such that the oscillatory behavior existing beyond the $\mathrm{SL}$ is transiently recreated. The second mechanism takes place close to the SNIC line, where the oscillatory behavior that is transiently recreated is that of the oscillations in region V. Both excitable behaviors exhibit a response starting at zero frequency (or infinite period), as both bifurcations are mediated by a saddle, whose stable manifold is the threshold beyond which perturbations must be applied to excite the system. In neuroscience terminology, both excitable behaviors are class (or type) I [35,36], although there are important differences between them. The SNIC mediated excitability is easier to observe than the one associated to a saddle-loop bifurcation for two reasons. First, it occurs in a broader parameter range due to its square-root scaling law (4), with respect to the SL excitability [37]. Second the excitable threshold can be controlled by the intensity of the localized Gaussian beam, that effectively approaches the fixed point and the saddle in phase space, allowing to reduce the threshold as much as desired (by approaching the SNIC line). Within region IV one can find a typical crossover behavior for the threshold, as it increases from zero (at the SNIC line) to the (finite) value characteristic of the SL bifurcation as one approaches this line.

Figure 6 shows the dynamics of the excitable fundamental solution in region IV, namely for the parameters corresponding to the fourth panel in Fig. 5, upon the application of different localized perturbations, one below the excitable threshold and two above. As expected, the perturbation below threshold relaxes directly to the fundamental solution 

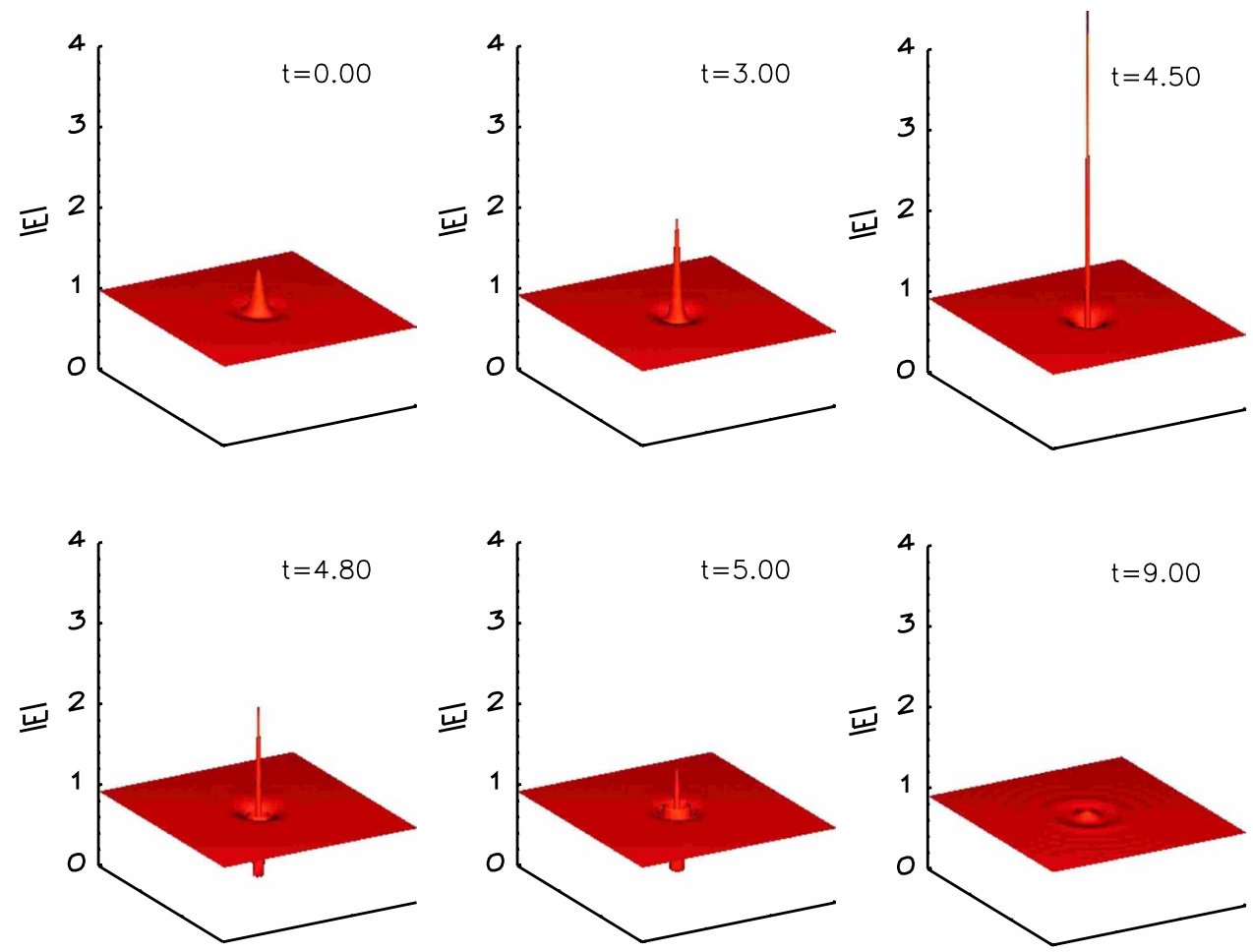

while the above threshold perturbations elicit first a large response of the system in the form of an excitable soliton which finally relaxes to the fundamental solution. The excitable excursion takes place at a later time as the smaller is the distance to the threshold (a signature of class I excitability). Finally, the shape of an excitable excursion in twodimensional space is shown in Fig. 7.

\section{CUSP CODIMENSION-2 POINT}

In the two-parameter phase diagrams in Figs. 2 and 3 one can see a point marked with a $C$ that was not found in the homogeneous case [38]. This point represents a cusp codimension-2 bifurcation point [39], namely a point in which two saddle-node curves merge. This cusp point, that involves only stationary (saddle-node) bifurcations, is also known as the cusp catastrophe [40]. For parameter values just at the left of the cusp the bump of the fundamental solution exhibits a rapid increase. Figure 8 shows the sharp, but smooth, change in the shape of the fundamental solution for three parameter values around the cusp point within region I. Instead, if one is to the right of the $C$ point this increase cannot be accommodated smoothly and a double fold occurs, such that three branches appear: Two stable, upper and lower branches, and one unstable, middle branch, and thus bistability makes its appearance. The two folds, codim-1, merge critically at the cusp point, codim-2. Decreasing $I_{s h}$ the cusp moves up towards $I_{s}=1$, so in the limit of homogeneous pump it cannot be seen due to the presence of the MI instability. The smooth connection between the fundamental branch and the upper branch exhibiting CS is an outcome of the symmetry breaking induced by the localized pump which has made the MI bifurcation become imperfect.

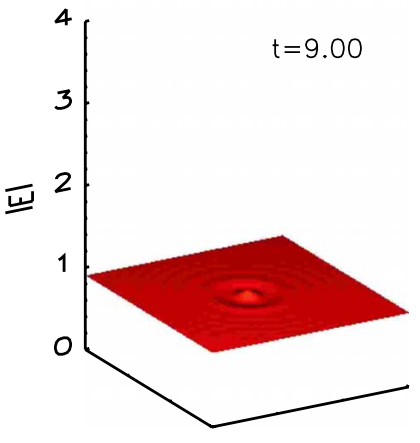

FIG. 7. (Color online) Transverse profile of $|E|$ at different times of the dotted line in Fig. 6.

\section{SADDLE-NODE SEPARATRIX-LOOP CODIMENSION-2 POINT}

The subject of the present section is to discuss the point designated with SNSL (that stands for saddle-node separatrix loop $[35,41]$ (also called saddle-node noncentral homoclinic bifurcation and saddle-node homoclinic orbit bifurcation [36]) in Figs. 2 and 3. An SNSL is a local-global codimension-2 point in which a saddle-node bifurcation takes place simultaneously to a saddle loop, such that the orbit enters through the noncentral (stable) manifold. The unfolding of a SNSL point leads to the scenario depicted in Fig. 9. There is a line of saddle-node bifurcations (in which a pair of stable and unstable fixed points are created) that at

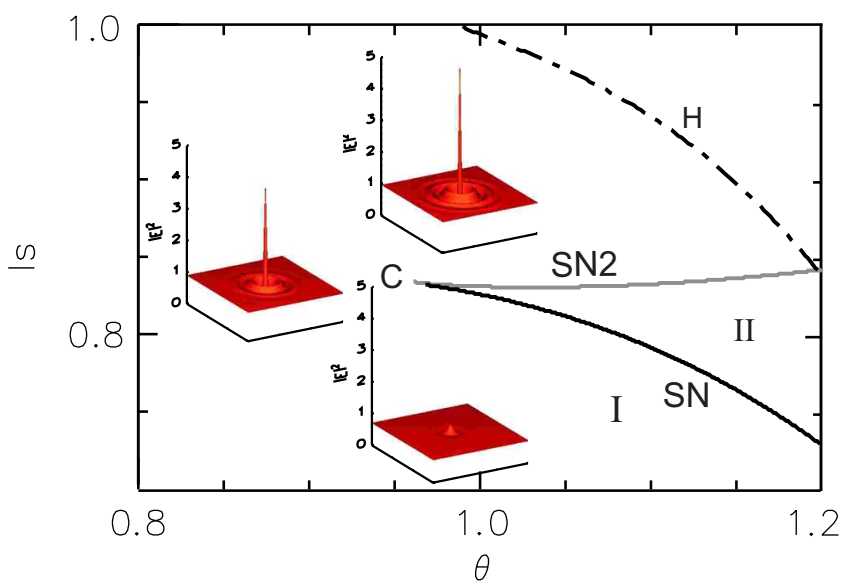

FIG. 8. (Color online) The fundamental solution for three points in the $\left(I_{s}, \theta\right)$ parameter space, for $I_{s h}=0.3$, around the cusp codimension- 2 point. The coordinates are (bottom to top) $I_{s}=0.7$, $\theta=0.9 ; I_{s}=0.85, \theta=0.85 ; I_{s}=0.95, \theta=0.95$. 


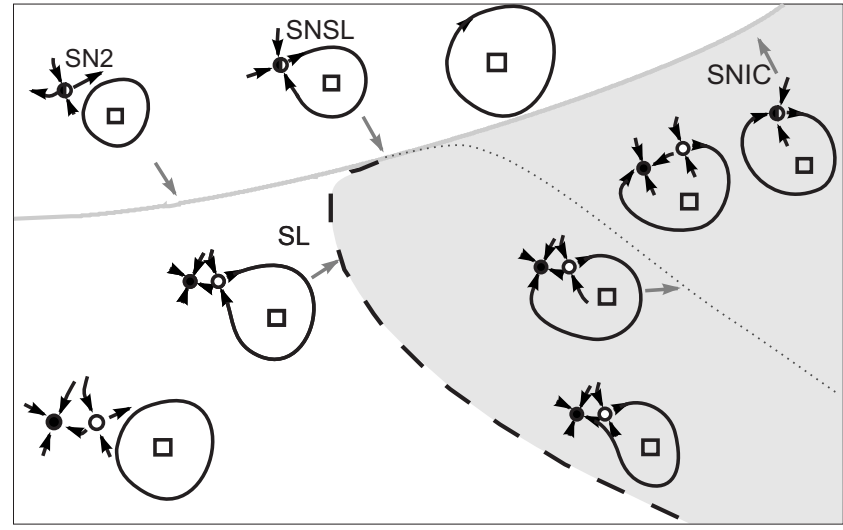

FIG. 9. Sketch of the parameter space near the SNSL point, showing the unfolding of bifurcation lines, and adapted to the geometry in Figs. 2 and 3.

one side of the SNSL is a saddle-node bifurcation off limit cycle (SN2) while at the other side is a SNIC bifurcation (the saddle-node occurs inside the limit cycle). A saddle-loop (SL) bifurcation also unfolds from the SNSL point, tangent to the SN2 line. From the other side, a special curve depicted with a dotted line in Fig. 9 should appear (cf. Fig. 22 in Ref. [35]). It is not a bifurcation line, but instead it is a special line in which the approach to the node point is through the most stable direction, namely the direction transverse to the central manifold at the SNIC-SN2 (and SNSL) bifurcations (for these reasons we also call this dotted line the pseudobifurcation line). This, in principle nongeneric, curve, that emerges in the unfolding of the SNSL point, is necessary for consistency on the excursions around the SNSL codimension- 2 point. At the pseudobifurcation the topological cycle is reconstructed, namely the stable fundamental solution and the middle branch CS (saddle), become points belonging to the circle that later will become the limit cycle. Before the pseudobifurcation, the unstable manifold of the saddle enters towards the stable solution from the same side as the saddle, while after the pseudobifurcation it enters from the opposite side.

The SNSL point separates two possible ways in which the system can go from oscillatory region III (where the limit cycle coexists with the stable fundamental solution) to oscillatory region $\mathrm{V}$ (where the fundamental solution does not exist).

For $\theta>\theta_{\text {SNSL }}$ the scenario is as described in Sec. IV, namely the middle and lower branches coalesce in a SNIC bifurcation. The main feature of this bifurcation is that it occurs on the limit cycle, leading to the excitable behavior of region IV. This scenario can be confirmed using the mode projection technique described in the Appendix of Ref. [24], that allows us to obtain in a quantitative form the phase space of an extended system (described, e.g., by a PDE), that strictly has an infinite dimension, but whose relevant dynamics is low dimensional. In this case, as in the case of a saddle-loop bifurcation studied in Ref. [24], we will argue that there are just two modes that are relevant, at least in the region close to the SNSL codimension-2 point. Figure 10 shows the spectrum of eigenvalues (linear stability analysis

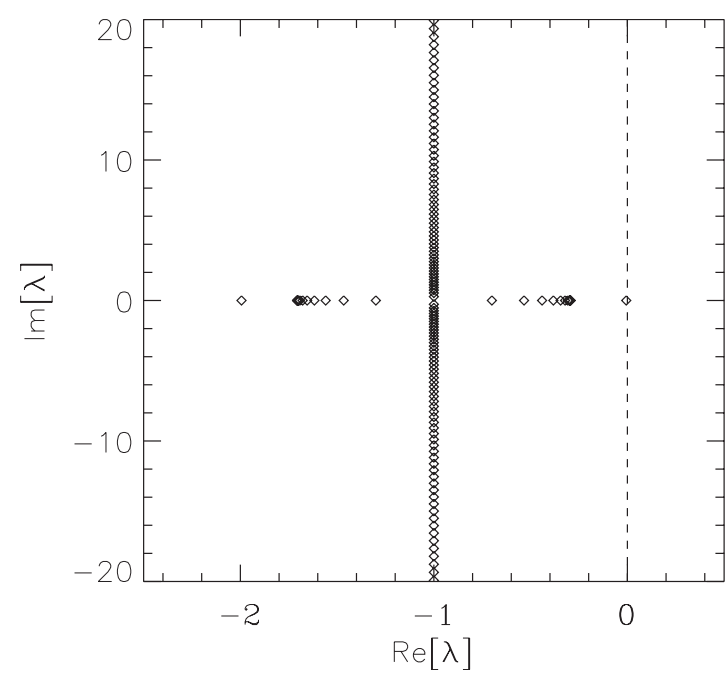

FIG. 10. Spectrum of the fundamental solution at the SNIC bifurcation for $I_{s h}=0.7, I_{s}=0.707$, and $\theta=1.34$ (close to the SNSL).

for the fundamental solution) at the SNIC bifurcation and close to the SNSL. The spectrum has a continuous part with eigenvalues lying along the line $\operatorname{Re}(\lambda)=-1$, and also a discrete part which is symmetric with respect to this line. It turns out that there are only two eigenmodes which are localized in space, while all the other eigenmodes are spatially extended. The two localized eigenmodes correspond to the most stable mode and to the one that becomes unstable. These two modes, shown in Fig. 11, are the only ones relevant for the dynamics of the CS close to the stable fixed point, since the projection of a localized solution onto any of the extended modes is negligible.

Figure 12 shows a quantitative reconstruction of the phase space. $\beta_{1}\left(\beta_{2}\right)$ corresponds to the amplitude of the projection of the trajectory along the unstable (most stable) eigenmode. Panel (a) represents a trajectory in the excitable region IV, close to the SNSL and below the pseudobifurcation line. Panel (b) shows a zoom of the trajectory close to the saddle (open circle) and the stable fundamental solution (filled circle). The excitable trajectory departs form the saddle and after long excursion in phase space arrives at the fundamental solution from below (that is, from the side of the saddle). Notice that while close to the fixed points the dynamics fit very well in a two-dimensional picture, away from them the line crosses, indicating that the full CS dynamics in phase space is not confined to a plane. Panel (c) corresponds to the pseudobifurcation, so that the trajectory arrives at the fundamental solution along the most stable direction. Panel (d) is just after the pseudobifurcation with the trajectory arriving from the other side. Finally panel (e) corresponds to parameters in the oscillatory region $\mathrm{V}$ just after the SNIC bifurcation. Notice that the pseudobifurcation line is very close to the SNIC bifurcation since we have taken parameters close to the SNSL and at the SNSL both lines originate tangentially. As expected, the quantitative picture agrees with the qualitative picture as one crosses the SNIC bifurcation in the right-hand panels of Fig. 5.

For $\theta<\theta_{\mathrm{SNSL}}$ the scenario is quite different, as the middle and lower branches now coalesce off the limit cycle, SN2, 

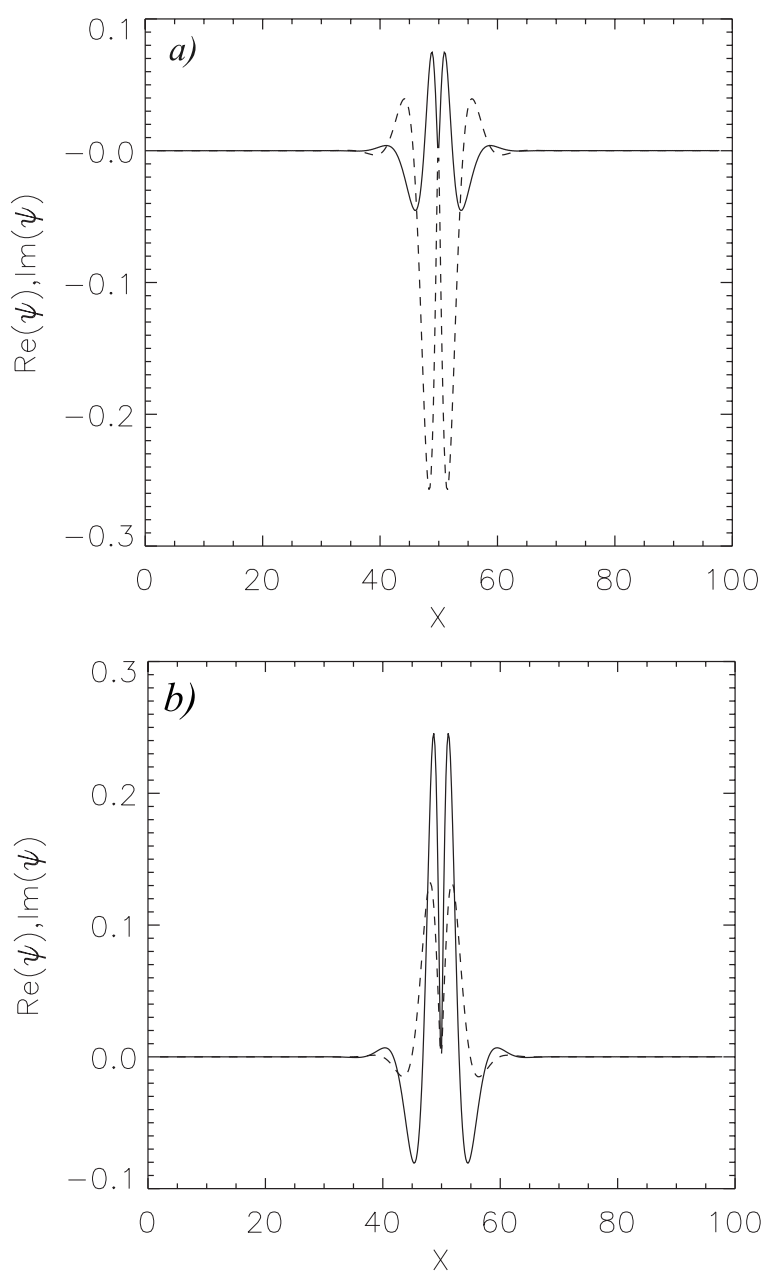

FIG. 11. Transverse cut of the most stable (a) and unstable (b) eigenmodes of the linear spectrum shown in Fig. 10. The solid (dashed) line indicates the real (imaginary) part of the eigenmode. which implies that the behavior of the system is oscillatory on both sides of the SN2 line, not excitable [42], and instead one crosses the SN2 line. This means that one is in a bistable oscillatory regime, and in crossing the SN2 line a saddlenode off the invariant cycle bifurcation occurs. SN2 involves the middle and fundamental branches (while SN involves the upper and middle branches). So, in the end this is nothing else than another way of entering region $\mathrm{V}$, of monostable oscillatory behavior, but instead of reconstructing the limit cycle, here the fundamental stationary solution is destroyed.

Therefore, and having in mind the behavior for $\theta \rightarrow \infty$ discussed in previous work for homogeneous pump [23,24], the overall scenario depicted in Figs. 2 and 3 is organized by three codimension- 2 points: a cusp point, from which two saddle-node bifurcations emerge (SN and SN2); an SNSL point from which a SNIC line emerges, and that organizes the SN2 and SL lines around; and a Takens-Bodgdanov point, occurring apparently at infinite detuning, where the SN, Hopf, and SL are tangent, and that can be seen as the birth of both the Hopf, and SL lines. The Takens-Bogdanov point was numerically shown to be present in the homogeneous case $[23,24]$. It is reassuring that the saddle-loop bifurcation line connects two of the codimension- 2 points, as it is not in some sense generic [43], and one does not expect that it emerges out of the blue. The scenario composed by these three codimension-2 bifurcations has been reported in other systems [44], and from a theoretical point of view, it can be shown that it appears in the unfolding in twodimensional parameter space of a codimension-3 degenerate Takens-Bogdanov point [45].

In the limit of homogeneous pump the SN2 and SNIC lines approach the MI line at $I_{s}=1$, which, therefore, also contains the cusp and the SNSL codimension-2 points. At the cusp the SN (responsible for the existence of CS) originates while at the SNSL the SL (originated at the TakensBogdanov and responsible of the CS excitability observed
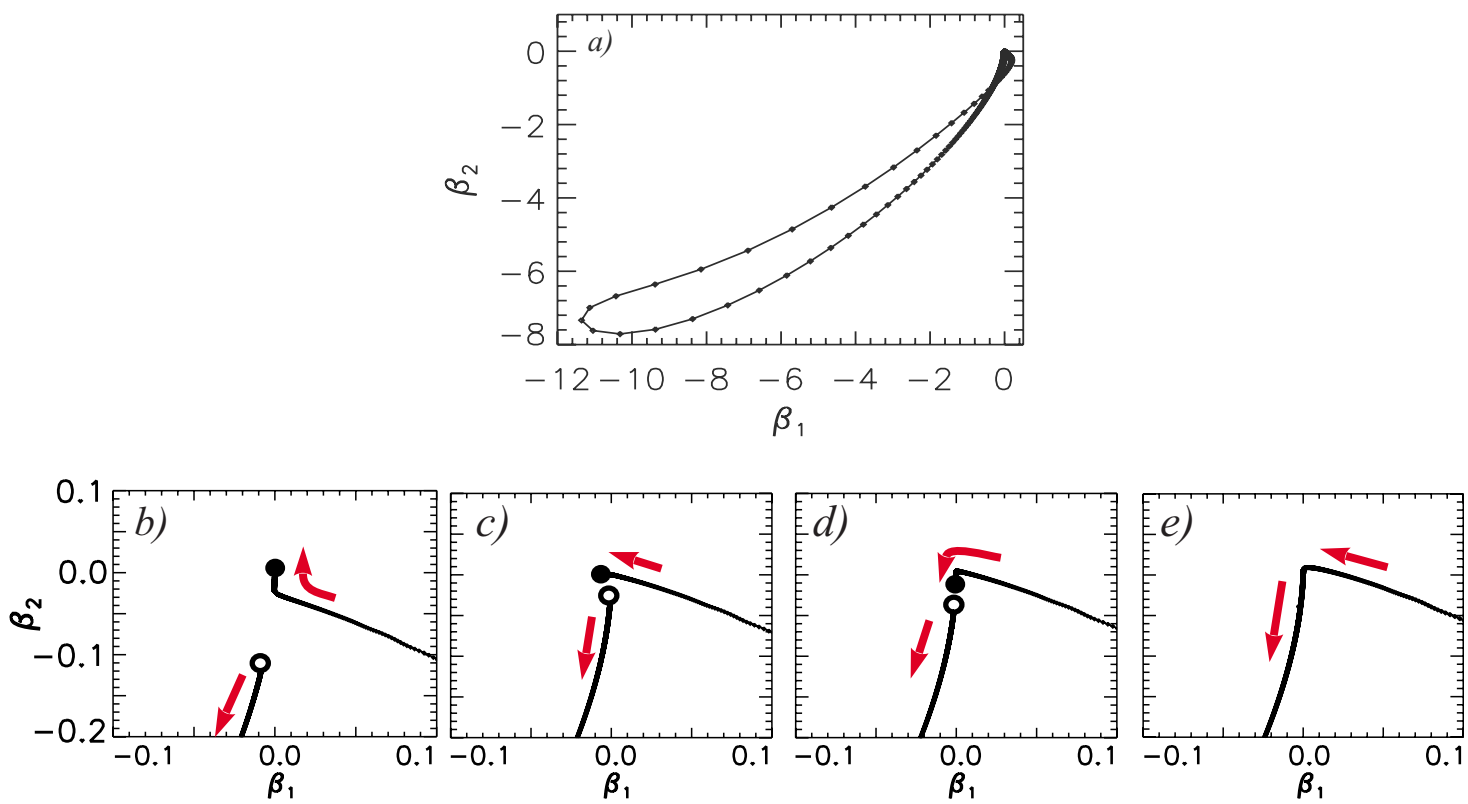

FIG. 12. (Color online) (a) Full trajectory in the phase space close to the SNIC $\left(I_{s}=0.863\right)$. The smaller panels show a zoom of the region in the phase space close to the fixed point. (b) $I_{s}=0.863$, (c) $I_{s}=0.8634575$, (d) $I_{s}=0.8635$, and (e) $I_{s}=0.864$. Here $I_{s h}=0.3$ and $\theta=1.34$. 
for homogeneous pump) ends. Notice that for homogeneous pump close to $I_{s}=1$ azimuthal instabilities renders the CS unstable to a pattern so it is difficult to study the SN and especially the SL lines in that region. Using a localized pump and then taking the limit to homogeneous pump circumvents these limitations.

\section{CONCLUSIONS}

We have presented a detailed study of the instabilities of solitons in nonlinear Kerr cavities under the application of a localized Gaussian beam. Since experimentally CS are typically switched on by applying a transient addressing beam the situation discussed here can be realized just by applying it on a permanent basis. The CS are sustained by a balance between nonlinearity and dissipation, as in the case of a homogeneous pump, although now these effects interact with the localized pump. The localized pump helps to spatially fix the CS and to control some of its dynamical properties. After the saddle-node bifurcation that creates the CS, it starts oscillating and overall exhibit a plethora of bifurcations that are shown to be organized by three codimension- 2 points: A Takens-Bogdanov point (which is also present for homogeneous pump as discussed already in $[23,24]$ ), a cusp, and a saddle-node separatrix loop (SNSL) points. In this scenario a saddle-loop bifurcation connects the Takens-Bogdanov and the SNSL and the cusp is connected to the other codimension- 2 bifurcations by two saddle-node lines. A line of SNIC bifurcations originates at the SNSL while at the
Takens-Bogdanov a Hopf bifurcation line meets tangentially a saddle-node and the saddle-loop lines.

The simultaneous presence in the system of two bifurcations that are associated to excitable behavior (saddle-loop and SNIC) enriches and completes the picture discussed for the case of a homogeneous pump. In fact the region for which excitable behavior is reported, in which the only attractor in the system is the fundamental solution, leads to two different class I behaviors (starting at infinite period). In the excitable region one goes smoothly from no threshold at the onset of the SNIC line to a finite threshold at the onset of the saddle-loop line. In fact, the excitable behavior mediated by a SNIC, and reported in this work, should be easier to observe both numerically and experimentally, and present some practical features that make it more suitable for practical applications. In particular, the excitable threshold can be controlled by the intensity of the localized beam. With an array of properly engineered beams, created, for instance, with a spatial light modulator, one could create reconfigurable arrays of coupled excitable units to all-optically process information. Work in this direction will be reported elsewhere.

\section{ACKNOWLEDGMENTS}

We acknowledge financial support from MEC (Spain) and FEDER (EU) through Grants No. FIS2007-60327 (FISICOS) and No. TEC2006-10009 (PhoDeCC), and from Govern Balear through Grant No. PROGECIB-5A (QULMI). We are grateful to Diego Pazó for useful discussions. A.J. acknowledges financial support from MEC.
[1] S. Koga and Y. Kuramoto, Prog. Theor. Phys. 63, 106 (1980).

[2] B. S. Kerner and V. V. Osipov, Autosolitons: A New Approach to Problems of Self-Organization and Turbulence (Kluwer, Dordrecht, 1994).

[3] V. K. Vanag and I. R. Epstein, Chaos 17, 037110 (2007).

[4] F. J. Niedernostheide, B. S. Kerner, and H. G. Purwins, Phys. Rev. B 46, 7559 (1992).

[5] P. B. Umbanhowar, F. Melo, and H. L. Swinney, Nature (London) 382, 793 (1996).

[6] J. J. Niemela, G. Ahlers, and D. S. Cannell, Phys. Rev. Lett. 64, 1365 (1990).

[7] O. Batiste and E. Knobloch, Phys. Rev. Lett. 95, 244501 (2005).

[8] O. Lejeune, M. Tlidi, and P. Couteron, Phys. Rev. E 66, 010901(R) (2002).

[9] W. J. Firth and C. O. Weiss, Opt. Photonics News 13, 55 (2002).

[10] Feature Section on Cavity Solitons, edited by L. A. Lugiato [IEEE J. Quant. Elect. 39(2) (2003)].

[11] N. N. Rosanov, Spatial Hysteresis and Optical Patterns, Springer Series in Synergetics (Springer, Berlin, 2002).

[12] S. Barland et al., Nature (London) 419, 699 (2002).

[13] Y. Tanguy, T. Ackemann, W. J. Firth, and R. Jäger, Phys. Rev. Lett. 100, 013907 (2008).

[14] M. Tlidi, M. Taki, and T. Kolokolnikov, Chaos 17, 037101
(2007).

[15] Dissipative Solitons, edited by N. Akhmediev and A. Ankiewicz, Lecture Notes in Physics, Vol. 661 (Springer, Berlin, 2005).

[16] Dissipative Solitons: From Optics to Biology and Medicine, edited by N. Akhmediev and A. Ankiewicz, Lecture Notes in Physics Vol. 751 (Springer, Berlin, 2008).

[17] P. Coullet, C. Riera, and C. Tresser, Chaos 14, 193 (2004).

[18] S. Fauve and O. Thual, Phys. Rev. Lett. 64, 282 (1990).

[19] M. Tlidi, P. Mandel, and R. Lefever, Phys. Rev. Lett. 73, 640 (1994).

[20] W. J. Firth, A. Lord, and A. J. Scroggie, Phys. Scr., T T67, 12 (1996).

[21] W. J. Firth, G. K. Harkness, A. Lord, J. McSloy, D. Gomila, and P. Colet, J. Opt. Soc. Am. B 19, 747 (2002).

[22] V. K. Vanag and I. R. Epstein, Phys. Rev. Lett. 92, 128301 (2004).

[23] D. Gomila, M. A. Matías, and P. Colet, Phys. Rev. Lett. 94, 063905 (2005).

[24] D. Gomila, A. Jacobo, M. A. Matías, and P. Colet, Phys. Rev. E 75, 026217 (2007).

[25] L. A. Lugiato and R. Lefever, Phys. Rev. Lett. 58, 2209 (1987).

[26] W. J. Firth and A. Lord, J. Mod. Opt. 43, 1071 (1996).

[27] J. Rasmussen and K. Rypdal, Phys. Scr. 33, 481 (1986). 
[28] D. Gomila and P. Colet, Phys. Rev. A 68, 011801(R) (2003).

[29] D. Gomila and P. Colet, Phys. Rev. E 76, 016217 (2007).

[30] S. H. Strogatz, Nonlinear Dynamics And Chaos (AddisonWesley, Reading, MA, 1994).

[31] When speaking about monostability and bistability we refer to localized solutions. In general, several extended solutions (patterns) may also be coexisting.

[32] P. Coullet, D. Daboussy, and J. R. Tredicce, Phys. Rev. E 58, 5347 (1998).

[33] M. Giudici, C. Green, G. Giacomelli, U. Nespolo, and J. R. Tredicce, Phys. Rev. E 55, 6414 (1997).

[34] D. Goulding, S. P. Hegarty, O. Rasskazov, S. Melnik, M. Hartnett, G. Greene, J. G. McInerney, D. Rachinskii, and G. Huyet, Phys. Rev. Lett. 98, 153903 (2007).

[35] E. M. Izhikevich, Int. J. Bifurcation Chaos Appl. Sci. Eng. 10, 1171 (2000).

[36] E. M. Izhikevich, Dynamical Systems in Neuroscience (MIT Press, Cambridge, MA, 2007).

[37] For saddle-loop mediated excitability the scaling law is logarithmic, implying that the frequency increases very fast from zero in a very narrow range, and, thus, its class I features can be easily missed experimentally [36].
[38] The cusp coordinates are $I_{s}=0.834, \theta=0.961$ for $I_{s h}=0.3$ (Fig. 2), and $I_{s}=0.61, \theta=0.968$ for $I_{s h}=0.7$ (Fig. 3).

[39] Y. A. Kuznetsov, Elements of Applied Bifurcation Theory, 3rd ed. (Springer-Verlag, Berlin, 2004).

[40] T. Poston and I. Stewart, Catastrophe Theory and its Applications (Longman, Essex, 1979).

[41] S. Schecter, SIAM J. Math. Anal. 18, 1142 (1987).

[42] Due to the bending of the SL line when approaching the SNSL, there is a region in which vertical paths in parameter space cross the SL line 2 times. Qualitatively there is no difference between crossing 2 times or none, as one is in the oscillatory bistable region III when approaching the SN2 line.

[43] Because it implies that a limit cycle has a tangency simultaneously with the stable and unstable manifolds of a saddle point.

[44] E. Meca, I. Mercader, O. Batiste, and L. Ramírez-Piscina, Theor. Comput. Fluid Dyn. 18, 231 (2004), and references therein.

[45] F. Dumortier, R. Roussarie, J. Sotomayor, and H. Zoladek, Bifurcations of Planar Vector Fields. Nilpotent Singularities and Abelian Integrals, Lecture Notes in Mathematics Vol. 1480 (Springer-Verlag, Berlin, 1991). 